\title{
Nernst effect in the electron-doped cuprate superconductors
}

\section{Citation}

Hackl, Andreas, and Subir Sachdev. 2009. "Nernst Effect in the Electron-Doped Cuprate Superconductors." Physical Review B 79 (23). https://doi.org/10.1103/physrevb.79.235124.

\section{Permanent link}

http://nrs.harvard.edu/urn-3:HUL.InstRepos:41412212

\section{Terms of Use}

This article was downloaded from Harvard University's DASH repository, and is made available under the terms and conditions applicable to Open Access Policy Articles, as set forth at http:// nrs.harvard.edu/urn-3:HUL.InstRepos:dash.current.terms-of-use\#OAP

\section{Share Your Story}

The Harvard community has made this article openly available.

Please share how this access benefits you. Submit a story.

\section{Accessibility}




\title{
Nernst effect in the electron-doped cuprates
}

\author{
Andreas Hackl ${ }^{1,2}$ and Subir Sachdev ${ }^{1}$ \\ ${ }^{1}$ Department of Physics, Harvard University, Cambridge, Massachusetts 02138, USA \\ ${ }^{2}$ Institut für Theoretische Physik, Universität zu Köln, Zülpicher Straße 77, 50937 Köln, Germany
}

(Dated: November 3, 2018)

\begin{abstract}
We calculate the normal state Nernst signal in the cuprates resulting from a reconstruction of the Fermi surface due to spin density wave order. An order parameter consistent with the reconstruction of the Fermi surface detected in electron-doped materials is shown to sharply enhance the Nernst signal close to optimal doping. Within a semiclassical treatment, the obtained magnitude and position of the enhanced Nernst signal agrees with Nernst measurements in electron-doped cuprates. Our result is mainly caused by the role of Fermi surface geometry under influence of a spin density wave gap. We discuss also possible roles of short-ranged magnetic order in the normal state Nernst effect and the Fermi surface reconstruction observed by photoemission spectroscopy.
\end{abstract}

\section{INTRODUCTION}

The Nernst effect has emerged as one of the key probes of the enigmatic underdoped phase of the cuprate high temperature superconductors. In the hole-doped case, observations 1 of a strongly enhanced Nernst signal at temperatures $(T)$ well above the superconducting $T_{c}$ have been interpreted ${ }^{1 / 2}$ using a picture of a liquid of vortices in the superconducting order. However, there have also been suggestions 3 that spin/charge density wave correlations of the vortex liquid are important. In particular, a model of fluctuations associated with the quantum phase transition (QPT) to the ordered stripe state at hole doping $\delta=1 / 8$ has been argued ${ }^{4}$ to have a Nernst response qualitatively similar to the observations.

In this paper, we focus on the electron-doped cuprates, where the situation appears simpler. The only observed order (apart from superconductivity) is a spin density wave (SDW) which remains commensurate at the $(\pi, \pi)$ wavevector (in the Brillouin zone of a square lattice of unit lattice spacing). The Nernst effect, being unmeasurable small in nearly all metals, has also been found to be anomalously large near optimal doping in the normal state of electron-doped cuprates $5 \mid 6$. We will show here that this large Nernst signal can be understood in a theory of Fermi surface reconstruction associated with the QPT involving onset of SDW order.

The large normal state Nernst signals found in $\mathrm{Pr}_{2-x} \mathrm{Ce}_{x} \mathrm{CuO}_{4-\delta}$ (PCCO) ${ }^{5}$ upon $\mathrm{Ce}$ doping, and in $\mathrm{Nd}_{2-x} \mathrm{Ce}_{x} \mathrm{CuO}_{4-\delta}$ (NCCO) upon oxygen doping, 6 have been attributed to the existence of two types of carriers, which avoid the Sondheimer cancellation of the Nernst signal expected in single carrier systems. Indeed, angle resolved photoemission spectroscopy (ARPES) experiments on NCCO found both electron- and hole-like Fermi pockets near optimal doping 7 . In the underdoped region, only small electron-like pockets remain, while in the overdoped region, only a large hole-like pocket centered at $(\pi, \pi)$ was found $\sqrt[8]{ }$. These features are believed to arise from the commensurate $(\pi, \pi)$ SDW order over a wide range of electron doping, as has been detected by various techniques $9|10| 11$. A possible critical doping for the SDW quantum critical point (QCP) has been inferred from transport measurements in the normal state, which show rapidly changing transport properties at $x_{c}=0.16512$. The assumption of a Fermi surface reconstruction caused by SDW order has led to a qualitative consistent description of Hall effect measurements on PCCO over a wide range of doping 13 .

It is important to note that there remain ambiguities about the critical value of doping where long-range magnetic order sets in. Elastic neutron scattering measurements on NCCO show that long-range magnetic order is preempted by short ranged antiferromagnetism for dopings between $x=0.134$ and $x=0.154^{14}$. It has still to be clarified whether short-ranged antiferromagnetism below optimal doping applies also to other electron-doped materials and is confirmed also by other techniques. We will argue that the main features of Fermi surface reconstruction observed in electron doped cuprates are induced by a true SDW gap. Especially in PCCO, there is no experimental evidence that magnetic order is short-ranged below optimal doping.

Our main result is that the related Nernst effect measurements on PCCO and NCCO can be explained by the emergence of hole-like carriers near optimal doping. These aspects will be quantified within a simple semiclassical Boltzmann approach.

\section{MODEL}

We consider electrons moving on a square lattice with dispersion

$$
\begin{aligned}
\varepsilon_{\mathbf{k}}= & -2 t_{1}\left(\cos k_{x}+\cos k_{y}\right)+4 t_{2} \cos k_{x} \cos k_{y} \\
& -2 t_{3}\left(\cos 2 k_{x}+\cos 2 k_{y}\right)
\end{aligned}
$$

and parameters $t_{1}=0.38 \mathrm{eV}, t_{2}=0.32 t_{1}$ and $t_{3}=$ $0.5 t_{2} \frac{15}{15}$, chosen to reproduce the Fermi surface measured in photoemission experiments 718 . We will focus on a carrier density corresponding to the electron-doped case, with a two-dimensional density $n=1+x>1$ per unit cell. Below critical doping $x_{c}=0.165$, we assume commensurate SDW order at wavevector $\mathbf{Q}=(\pi, \pi)$ 


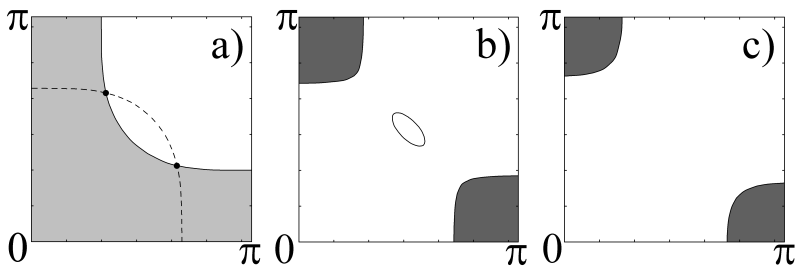

FIG. 1: Evolution of the Fermi surface upon decreasing electron doping $x$. To distinguish holes from electrons, electrons from the upper band $E_{\mathbf{k}}^{+}$are dark shaded, light shading contains all electrons from both bands. At $x=x_{c}=0.165$, a gap opens at the points where the dispersion crosses its translation by the wavevector $(\pi, \pi)$, see a). A hole pocket centered at $(\pi / 2, \pi / 2)$ is present for $x_{1}<x<x_{c}$ (with $x_{1}=0.145$ ), as shown in $\mathrm{b}$ ) for $x=0.15$. For $x<x_{1}$, only electron-like pockets remain, as shown in c) for $x=0.12$.

with scattering amplitude $\Delta^{1112}$. Microscopically, this order can be understood as a consequence of electronelectron interactions ${ }^{16}$, which are minimally described by the Hubbard interaction

$$
H_{e l-e l}=\frac{U}{N} \sum_{\mathbf{k}, \mathbf{k}^{\prime}, \mathbf{q}, \sigma} c_{\mathbf{k}, \sigma}^{\dagger} c_{\mathbf{k}+\mathbf{q}, \sigma} c_{\mathbf{k}^{\prime},-\sigma}^{\dagger} c_{\mathbf{k}^{\prime}-\mathbf{q},-\sigma} .
$$

The spin density wave instability is described by the complex order parameter

$$
\Delta e^{i \phi}=\frac{U}{N} \frac{1}{V} \sum_{\mathbf{k}}\left\langle c_{\mathbf{k} \uparrow}^{\dagger} c_{\mathbf{k}+\mathbf{Q}, \uparrow}\right\rangle=\frac{U}{N} \frac{1}{V} \sum_{\mathbf{k}}\left\langle c_{\mathbf{k} \downarrow}^{\dagger} c_{\mathbf{k}+\mathbf{Q}, \downarrow}\right\rangle,
$$

which can be determined self-consistently by employing the Hartree-Fock decoupling $c_{\mathbf{k}, \sigma}^{\dagger} c_{\mathbf{k}+\mathbf{q}, \sigma} \rightarrow$ $\left\langle c_{\mathbf{k} \sigma}^{\dagger} c_{\mathbf{k}+\mathbf{Q}, \sigma}\right\rangle \delta_{\mathbf{Q}, \mathbf{q}}$ in Eq. 2217 .

In the doubled unit cell and at mean field level, this changes the dispersion to

$$
E_{\mathbf{k}}^{ \pm}=\frac{1}{2}\left(\varepsilon_{\mathbf{k}}+\varepsilon_{\mathbf{k}+\mathbf{Q}} \pm \sqrt{\left(\varepsilon_{\mathbf{k}}-\varepsilon_{\mathbf{k}+\mathbf{Q}}\right)^{2}+4 \Delta^{2}}\right),
$$

where now the reduced antiferromagnetic Brillouin zone has to be considered. The quasiparticles resulting from the reconstructed bands have the velocities

$$
v_{\mathbf{k}}^{ \pm}=\frac{1}{\hbar} \nabla_{\mathbf{k}} E_{\mathbf{k}}^{ \pm} / \hbar
$$

where we will omit an explicit band label from the velocities in the following in order to compactify our notation. Consistent with the Hartree-Fock treatment of the effective Hamiltonian, we chose a mean field dependence $\Delta(x)[e V]=0.7 \sqrt{1-x / 0.165}$. The gap opens rapidly on depleting the carrier concentration below $x_{c}=0.165$ and the Fermi surface reconstructs in qualitative agreement with ARPES data ${ }^{7 / 8}$, see Fig. 1.

A gap of $\Delta=0.7 \mathrm{eV}$ yields also consistent results for the Hall coefficient 13 . Our results are not sensitive to precise parameter choices, and slight variations of parameters lead only to minor modifications of our results. We will show that the opening of a hole pocket will strongly influence the Nernst effect. Moreover, our modeling agrees with Hall measurements by Onose et al $\frac{19}{19}$, which indicate that the hole pockets are present for $x_{1}<x<x_{c}$ Ce doping with $x_{1}=0.1$.

\section{SEMICLASSICAL APPROACH}

Several parameter scales have to be set to justify our Boltzmann approach. Backscattering of the SDW amplitude sets a momentum scale $p_{\Delta}=\Delta / v_{F}\left(v_{F}\right.$ is the Fermi velocity), while the inverse mean free path $l^{-1}$ defines another momentum scale. To neglect interference effects between scattering events, the momentum scale $p_{0}$ set by the size of the Brillouin zone has to fulfill $p_{0} \gg p_{\Delta}, l^{-1}$ 13. At low $T$, we assume that impurity scattering dominates the relaxation time $\tau$. In general, cuprate materials show a normal state quasiparticle scattering rate which is linear in temperature, with a small part of the antinodal region where there is no temperature dependence observed ${ }^{20}$. We will neglect effects of anisotropy and temperature on the scattering rate by assuming pure s-wave impurity scattering, as is appropriate for randomly distributed impurities with weak and shortranged scattering potential. Lateron, we will return to possible modifictations due to scattering anisotropy and thermal fluctuations.

Disorder is expected to modify the SDW backscattering if the mean free path $l$ drops below the characteristic scattering length on the SDW order parameter. This situation is expected to occur if $p_{\Delta} l \sim 1$, and we will consider only $\Delta>v_{F} / l$. Finally, weak magnetic fields make it possible to expand transport coefficients in magnetic field strength, so that off-diagonal transport coefficients become linear in $B$, while the diagonal coefficients are independent of $B$. The applicability of this expansion is related to the momentum scale set by $a=\pi l B / \phi_{0}$ with the flux quantum $\phi_{0}=h c / 2 e$, which defines the weak-field regime $a<p_{\Delta} \underline{13}$, where the Zener-Jones expansion is applicable. Magnetic fields also have to be weak enough to neglect magnetic breakdown. Neglecting modifications of magnetic field on the band structure, magnetic breakdown is analogous to Zener breakdown and has a transmission amplitude $\mathrm{e}^{21}$

$$
\alpha=\exp \left(-\frac{\pi}{2} \frac{\Delta^{2}}{e \hbar B\left|v_{x} v_{y}\right|}\right)
$$

with the Fermi velocities $v_{x}, v_{y} \approx v_{F}$ of the linearized dispersion at its crossing point obtained by setting $\Delta=0$. Therefore, magnetic breakdown can be neglected as long as $p_{\Delta}>p_{B}$, where the inverse magnetic length $p_{B}=$ $2 \pi\left(\pi B / \Phi_{0}\right)^{-1 / 2}$ appears.

In mean-field approximation, the transport processes are determined by the current operator

$\mathbf{j}=-e \sum_{\sigma} \int_{R B Z} \frac{d^{2} \mathbf{k}}{(2 \pi)^{2}} \psi_{\mathbf{k}, \sigma}^{\dagger}\left(\begin{array}{cc}\nabla_{\mathbf{k}} E_{\mathbf{k}}^{+} / \hbar & v_{\mathbf{k}}^{\text {inter }} \\ v_{\mathbf{k}}^{\text {inter }} & \nabla_{\mathbf{k}} E_{\mathbf{k}}^{-} / \hbar\end{array}\right) \psi_{\mathbf{k}, \sigma}$, 
where the spinor $\psi_{\mathbf{k}, \sigma}$ contains the two quasiparticle modes. The current therefore includes also scattering events between bands mediated by the off-diagonal elements

$$
v_{\mathbf{k}}^{\text {inter }}=-\frac{1}{\hbar} \frac{\left[\nabla_{\mathbf{k}} \epsilon_{\mathbf{k}}-\nabla_{\mathbf{k}} \epsilon_{\mathbf{k}+\mathbf{Q}}\right] \Delta}{\sqrt{\left(\epsilon_{\mathbf{k}}-\epsilon_{\mathbf{k}+\mathbf{Q}}\right)^{2}+4 \Delta^{2}}}
$$

However, if the energy gap to the second band is larger than $k_{B} T$ and $\hbar / \tau$, interband contributions to transport can be neglected. We will neglegt a small doping range very close to the $\mathrm{QCP}$, where $p_{\Delta}$ might be small enough to allow for magnetic breakdown or modifications due to disorder. For magnetic fields of order a few Tesla and scattering times of $\mathcal{O}\left(10^{-14} s\right)$, this doping range is expected to be difficult to detect in experiment. According to formula (6), magnetic breakdown is of importance in the doping range $\Delta x \approx e \hbar v_{F}^{2} B /(0.7 e V)^{2} x_{c} \approx$ $7.1 \times 10^{-5} B x_{c}$, where we used the universal Fermi velocity $v_{F}=2.3 \times 10^{7} \mathrm{~cm} / \mathrm{s}^{22}$. Interband transitions mediated by impurity scattering are estimated to occur in the doping range

$$
\Delta x \approx\left(\frac{\hbar}{\tau}\right)^{2} \frac{x_{c}}{(0.7 e V)^{2}} \approx 8.6 \times 10^{-3}\left(\frac{10^{-14} s}{\tau}\right)^{2} x_{c},
$$

which is negligible for relaxation times of $\mathcal{O}\left(10^{-14} s\right)$. From experimental data at optimal doping, the relaxation time can be estimated to be somewhat larger than $10^{-14} s$, see below. Assuming an ordering temperature $T_{S D W}=T_{0} \sqrt{1-x / x_{c}}$ with $T_{0} \approx 250 K^{23}$, thermal excitations destroy the SDW gap in a range of width $\Delta x \approx x_{c}\left(T / T_{0}\right)^{2}$ below doping $x_{c}$. Keeping this in mind, we assume that all mentioned considerations are valid for the parameter regimes discussed below.

We define the thermoelectric response in the absence of an electrical current as

$$
\mathbf{E}=-\hat{\vartheta} \vec{\nabla} T
$$

from which the Nernst signal $e_{N}=\vartheta_{y x}$ and the thermoelectric power $Q=\vartheta_{x x}$ are obtained. For square lattice geometry, the diagonal entries of all transport tensors are isotropic. Both coefficients can be expressed as

$$
\begin{aligned}
& \vartheta_{y x}=\frac{\alpha_{x y} \sigma_{x x}-\alpha_{x x} \sigma_{x y}}{\sigma_{x x}^{2}+\sigma_{x y}^{2}} \\
& \vartheta_{x x}=\frac{\alpha_{x x}}{\sigma_{x x}},
\end{aligned}
$$

where the usual definitions of the electrical and thermoelectrical conductivities enter 24 . To calculate the quasiparticle Nernst signal, we restrict us to the weak-field regime defined above. From the linearized Boltzmann equation, we obtain the transport coefficients ${ }^{24}$

$$
\begin{aligned}
& \alpha_{x x}=\frac{2 e}{T} \sum_{\mathbf{k}, \alpha= \pm} \frac{\partial f_{\mathbf{k}}^{0}}{\partial E_{\mathbf{k}}^{\alpha}}\left(E_{\mathbf{k}}^{\alpha}-\mu\right) \tau_{\mathbf{k}}\left(v_{\mathbf{k}}^{x}\right)^{2} \\
& \alpha_{x y}=\frac{2 e^{2} B}{T \hbar c} \sum_{\mathbf{k}, \pm} \frac{\partial f_{\mathbf{k}}^{0}}{\partial E_{\mathbf{k}}^{\alpha}}\left(E_{\mathbf{k}}^{\alpha}-\mu\right) \tau_{\mathbf{k}}^{2} v_{\mathbf{k}}^{x}\left[v_{\mathbf{k}}^{y} \frac{\partial v_{\mathbf{k}}^{y}}{\partial k_{x}}-v_{\mathbf{k}}^{x} \frac{\partial v_{\mathbf{k}}^{y}}{\partial k_{y}}\right] \\
& \sigma_{x x}=-2 e^{2} \sum_{\mathbf{k}, \pm} \frac{\partial f_{\mathbf{k}}^{0}}{\partial E_{\mathbf{k}}^{\alpha}} \tau_{\mathbf{k}}\left(v_{\mathbf{k}}^{x}\right)^{2} \\
& \sigma_{x y}=-2 \frac{e^{3} B}{\hbar c} \sum_{\mathbf{k}, \pm} \frac{\partial f_{\mathbf{k}}^{0}}{\partial E_{\mathbf{k}}^{\alpha}} \tau_{\mathbf{k}}^{2} v_{\mathbf{k}}^{x}\left[v_{\mathbf{k}}^{y} \frac{\partial v_{\mathbf{k}}^{y}}{\partial k_{x}}-v_{\mathbf{k}}^{x} \frac{\partial v_{\mathbf{k}}^{y}}{\partial k_{y}}\right], \quad(12
\end{aligned}
$$

where $\alpha= \pm$ denotes summation over the quasiparticle bands of Eq. (4). For brevity, we have droped the band index from the quasiparticle velocities, which have been properly defined in Eq. (5). It will be of interest to study Eq. (12) in dependence of electron doping in order to analyze the influences of Fermi surface changes on transport properties. At low $T$, the thermoelectric conductivities $\alpha_{i j}$ are related to the electrical conducticities $\sigma_{i j}$ by the Mott relation

$$
\alpha_{i j}=-\left.\frac{\pi^{2}}{3} \frac{k_{B}^{2} T}{e} \frac{\partial \sigma_{i j}}{\partial \mu}\right|_{E_{F}} .
$$

As long as the relaxation time depends on energy, the expression $\left.\frac{\partial \sigma_{i j}}{\partial \mu}\right|_{E_{F}}$ contains a contribution

$$
\frac{\left.\frac{\partial \tau}{\partial \mu}\right|_{E_{F}}}{\tau}\left(2-\delta_{i j}\right) \sigma_{i j}
$$

\section{A. Comparison with experiment}

Due to Eq. 137, the energy dependence $\partial \tau / \partial \mu$ enters thermoelectric quantities. We rule these contributions out by using a constant $\tau$, in order to focus on the role of Fermi surface geometry in the Nernst effect. Usually, the energy dependence of $\tau$ is expected to behave as $\tau \propto E^{p}$, with $p \in[-1 / 2,3 / 2] 25$. In the low temperature regime, according to Fermi's golden rule $\tau \propto 1 / N(\epsilon)$, with $p=0$ for the two dimensional Fermi gas. Phonon contributions become only of importance for $T \gtrsim \Theta_{D}$, for which $\mathrm{p}=3 / 2$. Thus, phonons lead to a positive contribution in Eq. (14). In two dimensions, energy dependence of the relaxation time due to impurities yields corrections to the Nernst signal which vanish in the free electron case, making them sensitively dependent on details of the band structure.

We estimated these effects numerically by setting $\tau^{\prime} \equiv$ $(\partial \tau / \partial \mu)_{E_{F}}=\tau / E_{F}$, which yields a negligible correction to the peak signal, see Fig. 2. On the other hand, if $\partial \tau / \partial \mu$ would contribute considerably to the Nernst signal, employing Eq. (13) in Eq. (11) shows that $\vartheta_{y x}=\mathcal{O}\left(\vartheta_{x x} \tan \left(\Theta_{H}\right)\right)$ with $\tan \left(\Theta_{H}\right)=\sigma_{x y} / \sigma_{x x}$. However, Nernst measurements on PCCO clearly show 
$\vartheta_{x x} \tan \left(\Theta_{H}\right) \ll \vartheta_{y x}$ for all Ce concentrations $x>0.05^{55}$, and we can thus neglect $\partial \tau / \partial \mu$.

We solved Eq. 12 numerically in the regime where $\vartheta_{y x}$ and $\vartheta_{x x}$ depend linearly on $T$, as shown in Fig. 2, The experimental peak height near optimal doping is reproduced in order of magnitude by the experimental value $\tau=3.30 \times 10^{-14} s^{-1}$ at optimal doping, which is obtained from the residual ab-plane resistivity $\rho=57 \mu \Omega \mathrm{cm}^{12}$ and the plasma frequency $\omega_{p}=13000 \mathrm{~cm}^{-1}[26$. In a range above optimal doping, the peak structure of the experimental signal is comparable with our theory. The experimental Nernst signal seems to be shifted by $\Delta x \approx 0.02$ on the doping axis, suggesting that the carrier concentration of the sample differs from nominal doping by the same amount. A deviation of $2 \%$ carrier concentration is quantitatively also found in a comparison of the Fermi volume found from ARPES and the Fermi volume calculated from Eq. 11 27. In addition, a calculation of the Hall coefficient in dependence of electron doping using the dispersion of Eq. (1) shows also a shift of about $2 \%$ carrier concentration with respect to experimental results in the underdoped regime, which also fail to reproduce the expected $R_{H} \propto 1 / x$ behavior if $x$ is set equal to the Ce concentration 13 . The deviation could be caused by high $T$ oxygen annealing, which leads to doping inhomogeneity/uncertainty in large crystals 28 .

We therefore interpret the peak in the Nernst measurements near optimal doping as a result of an emerging hole pocket. A related enhancement of the Nernst signal near van Hove singularities has been described by Livanov 29 . The Nernst signal further away from optimal doping is not accurately reproduced by our model; anisotropy of the scattering rate ${ }^{20}$ is a possible origin of the sizable signal, and scattering off order parameter fluctuations should also be considered 13 .

The relaxation time approximation (RTA) is a doubtful method to reproduce the influence of antiferromagnetic fluctuations on transport properties. In RTA, the quasiparticle current is given by $J_{\mathbf{k}}=\tau_{\mathbf{k}} v_{\mathbf{k}}$, thereby neglecting current vertex corrections $\Delta J_{\mathbf{k}}$ caused by the interaction-induced drag of surrounding quasiparticles. These corrections are important to maintain a conserving approximation in the sense of Kadanoff and Baym ${ }^{30}$. The influence of antiferromagnetic fluctuations on the Nernst signal is more accurately treated within the FLEX+tmatrix approximation 3 , which is beyond the scope of this paper. We will analyze corrections due to antiferromagnetic fluctuations at low temperatures more detailed in section IV.

\section{B. Nernst effect near singular doping}

The behavior of the Nernst coefficient near the singular dopings in Fig. 2 can be obtained from analytical considerations. First, we consider the singularity at doping $x_{1}$, where hole like carriers emerge in the Fermi volume, see Fig. 1. The hole pocket corresponds to a

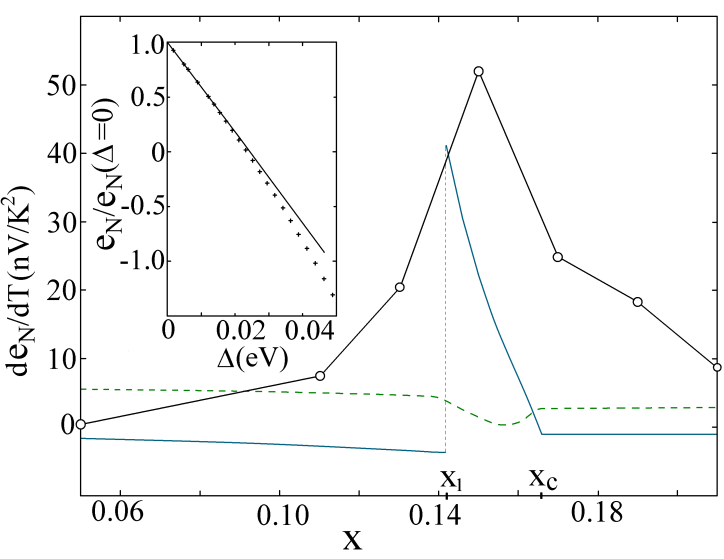

FIG. 2: Dependence of the Nernst coefficient on electron doping in the limit $T \rightarrow 0$. With decreasing $x$, the coefficient has an onset near $x=x_{c}$, where SDW order sets in; The discontinuity at $x=x_{1}$ is due to the opening of hole pockets (blue curve). The magnitude of our estimate of contributions due to energy dependence of the relaxation time has negligible size in the peak region (dashed line), as compared to the experimental values (black curve). Experimental data points from Ref. 5 correspond to the small circles, the line is a guide to the eye. The inset shows the quantum critical contribution to $\vartheta_{y x}$, which becomes large already at small gap energies $\Delta$. Numerical data points in the inset correspond to the crosses, which asymptotically behave as a linear function of gap amplitude, as given by the black line.

local minimum of the dispersions Eq. (4), whose distance from the chemical potential can be expanded as $\Delta E=(d \mu / d x)_{x_{1}}\left(x-x_{1}\right)+\mathcal{O}\left(x-x_{1}\right)^{2}$, as we confirmed numerically. Analogous to the discussion in the context of the SDW gap, the gap energy $\Delta E$ has to be large enough in order to neglect magnetic breakdown and thermal excitations across the gap. These effects tend to smear out the discontinuity in the Nernst signal over a finite range of doping, while the order of magnitude in change in the signal is not expected to change considerably.

The asymptotic low temperature limit of the Sommerfeld expansion is valid as long as $k_{B} T \ll|\Delta E|$, and thus thermal excitations change the behavior near the hole pocket in a finite range $\Delta x \approx\left(k_{B} T\right) /|d \mu / d x|_{x_{1}}$ of doping. Considering the numerical value $d \mu /\left.d x\right|_{x_{1}} \approx 1.52 \mathrm{eV}$, $\Delta x<0.01$ at $T<100 \mathrm{~K}$. An estimate of the doping range where magnetic breakdown according to formula (6) can occur is given by

$$
\Delta x \approx \frac{v_{F}}{\left|\frac{d \mu}{d x}\right|_{x_{1}}} \sqrt{\frac{2 \hbar e}{\pi} B} \approx 5 \times 10^{-3} \sqrt{B}
$$

where the universal Fermi velocity $v_{F}=2.3 \times 10^{7} \mathrm{~cm} / \mathrm{s}$ and the numerical value $\left|\frac{d \mu}{d x}\right|_{x_{1}} \approx 1.52 \mathrm{eV}$ have been used. For experimentally relevant magnetic field strengths of $B \approx 10 T$, the Nernst signal is therefore expected to become sharply enhanced already for dopings of about 
$1-2 \%$ below $x_{1}$, consistent with the experimental result shown in Fig. 2 ,

Near the opening of the hole pocket at $x=x_{1}$, the hole dispersion is approximated by $\varepsilon_{h}(\mathbf{k})=\sum_{i} \delta k_{i}^{2} / m_{i}-\mu_{h}$, and the $T=0$ hole contributions to electrical transport become

$$
\begin{aligned}
\sigma_{x x}^{h}\left(\mu_{h}\right) & =\frac{2}{3} \mu_{h} \tau_{h}\left(\mu_{h}\right) e^{2} \frac{N_{h}}{\bar{m}_{h}} \\
\sigma_{x y}^{h}\left(\mu_{h}\right) & =\frac{2}{3} \mu_{h} \tau_{h}^{2}\left(\mu_{h}\right) \frac{e^{3} B}{c} \frac{N_{h}}{\bar{m}_{h}}
\end{aligned}
$$

for $\mu_{h}>0$ and vanish otherwise. In the following, we formally distinguish electron and hole scattering rates. The hole DOS $N_{h}$ and the reduced hole mass $\bar{m}_{h}=$ $\left(m_{1} m_{2}\right) /\left(m_{1}+m_{2}\right)$ are taken to be constant. For weak dilute disorder, the scattering rate follows $1 / \tau\left(\mu_{h}\right) \propto N_{h}$ and is energy independent. According to Eqs (13) and (16), the Nernst signal and the thermopower have discontinuities at $\mu_{h}=0$

$$
\begin{aligned}
& \Delta \vartheta_{y x}=\left[\frac{\sigma_{x x}^{e} \alpha_{x y}^{h}-\sigma_{x y}^{e} \alpha_{x x}^{h}}{\left(\sigma_{x x}^{e}\right)^{2}}\right]_{\mu_{h}=0^{+}} \\
& \Delta \vartheta_{x x}=\left[\frac{\alpha_{x x}^{h}}{\sigma_{x x}^{e}}\right]_{\mu_{h}=0^{+}} .
\end{aligned}
$$

Expanding the electron dispersion as $\varepsilon(\mathbf{k})=$ $\sum_{i} \delta k_{i}^{2} / m_{i}-\mu$, the relative changes are

$$
\begin{aligned}
\frac{\Delta \vartheta_{y x}}{\left.\vartheta_{y x}\right|_{\mu_{h}=0^{-}}} & =-\frac{N_{h} \bar{m}_{e} \tau_{h}}{N_{e} \bar{m}_{h} \tau_{e}}\left[\frac{\tau_{h}+\tau_{e}}{\tau_{e}^{\prime} \mu_{e}}\right] \\
\frac{\Delta \vartheta_{x x}}{\left.\vartheta_{x x}\right|_{\mu_{h}=0^{-}}} & =-\frac{\tau_{h} N_{h} \bar{m}_{e}}{\tau_{e} N_{e} \bar{m}_{h}} .
\end{aligned}
$$

Sizable contributions from the discontinuity can therefore be expected, and $\tau_{e}^{\prime}<0$ would explain why the Nernst signal shows no sign change in experiments on $\mathrm{PCCO}^{5}$. Moreover, a sign change near $x=0.15$ has been found in the thermoelectric power 11 , as predicted by Eq. (18). Assuming $\tau_{h} N_{h} \bar{m}_{e} \approx \tau_{e} N_{e} \bar{m}_{h}$, the magnitude of the discontinuity in the thermopower is about twice the magnitude of the thermoelectric power in the overdoped region. This relative change in thermopower is quantitatively equivalent to the change observed from $x=0.15$ to $x=0.16$ in the thermopower measurements from Ref. 11. We briefly extend this analysis to a general two-carrier system with carrier types 1 and 2, where $\Delta \vartheta_{y x}=\left(\sigma_{x x}^{(1)} \alpha_{x y}^{(2)}-\sigma_{x y}^{(1)} \alpha_{x x}^{(2)}\right) /\left(\sigma_{x x}^{(1)}\right)^{2}$ right at the emergence of carrier type 2, since Eq. 16 leads to $\hat{\sigma}^{(2)}=0$ at the opening of a carrier pocket. According to Eq. (13) and considering positive magnetic field strenghts $B$ in the following, $\alpha_{x y}^{(2)}$ is always positive and $\alpha_{x x}^{(2)}$ has always the sign of $\sigma_{x y}^{(2)}$ due to Eq. 13. This means that $\Delta \vartheta_{y x}$ is always positive if the carriers 1 and 2 have opposite charge, while $\Delta \vartheta_{y x}$ might both be negative or positive if carrier type 1 and 2 have the same charge. To decide on the charges of carriers 1 and 2, in addition the sign of the second contribution in $\Delta \vartheta_{y x}$ can be determined from a measurement of $\Delta \vartheta_{x x} \tan \left(\Theta_{H}\right)$.

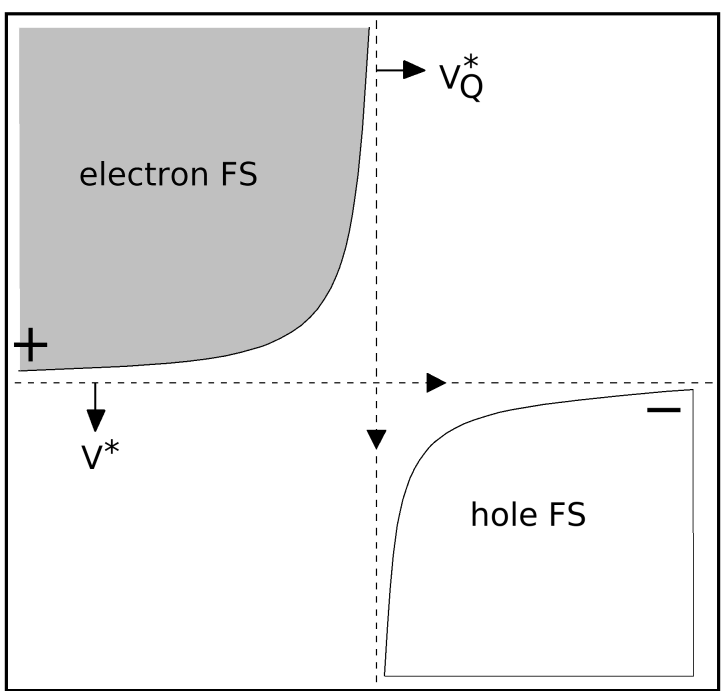

FIG. 3: To leading order in the gap amplitude $\Delta$, opening of the SDW gap modifies the Fermi surface only near the crossing points in momentum space where $\varepsilon_{\mathbf{p}}=\varepsilon_{\mathbf{p}+\mathbf{Q}}=\mu$. As shown in this sketch, a crossing point is coinciding with the crossing of the dashed lines as long as curvature of the Fermi surfaces is neglected near the crossing point. The vertical dashed line is the Fermi line for the normal state which is parallel to the vector $(0, \pi)$. The horizontal dashed line is the normal state Fermi line shifted by $\mathbf{Q}=(\pi, \pi)$, thus directing parallel to $(\pi, 0)$. The reconstructed Fermi surface contains electron pockets, denoted by + , and hole pockets, denoted by $-$

\section{Behavior near quantum critical point}

We now analyze the onset of the Nernst signal at the $x=x_{c}$ QCP where $\Delta$ first becomes non-zero with decreasing $x$. A calculation analogous to Refs 1332 can be employed to calculate the change $\delta \vartheta=\vartheta(\Delta)-\vartheta(\Delta=0)$ to linear order in the gap $\Delta$. The changes of the dispersion to leading order in $\Delta$ occur around momenta $\mathbf{p}$ with $\epsilon_{\mathbf{p}+\mathbf{Q}}=\epsilon_{\mathbf{p}}=\mu$, which are given by the crossing points in Fig. 1 $1 \mathrm{a}$ and their symmetry related counterparts. It is useful to parametrize $\mathbf{p}$ by $\varepsilon_{\mathbf{p}}$ and $\varepsilon_{\mathbf{p}+\mathbf{Q}}$, what is possible in the vicinity of any crossing point $\mathbf{p}^{\star}$. This can be achieved by expanding the dispersions

$$
\begin{aligned}
\varepsilon_{\mathbf{p}}-\mu & =\mathbf{v}^{\star} \cdot \delta \mathbf{p}+\frac{m_{i j}}{2} \delta p_{i} \delta p_{j} \\
& +\frac{y_{i j k}}{6} \delta p_{i} \delta p_{j} \delta p_{k}+\mathcal{O}\left(\delta p^{4}\right) \\
\varepsilon_{\mathbf{p}+\mathbf{Q}}-\mu & =\mathbf{v}_{\mathbf{Q}}^{\star} \cdot \delta \mathbf{p}+\frac{n_{i j}}{2} \delta p_{i} \delta p_{j} \\
& +\frac{z_{i j k}}{6} \delta p_{i} \delta p_{j} \delta p_{k}+\mathcal{O}\left(\delta p^{4}\right)
\end{aligned}
$$


where

$$
\begin{aligned}
\delta \mathbf{p} & =\mathbf{p}-\mathbf{p}^{\star} \\
\mathbf{v}^{\star} & =\mathbf{v}\left(\mathbf{p}^{\star}\right), \quad \mathbf{v}_{\mathbf{Q}}^{\star}=\mathbf{v}\left(\mathbf{p}^{\star}+\mathbf{Q}\right) \\
m_{i j} & =\left.\left(\partial^{2} \varepsilon_{\mathbf{p}} / \partial p_{i} \partial p_{j}\right)\right|_{\mathbf{p}^{\star}} \\
n_{i j} & =\left.\left(\partial^{2} \varepsilon_{\mathbf{p}} / \partial p_{i} \partial p_{j}\right)\right|_{\mathbf{p}^{\star}+\mathbf{Q}} \\
y_{i j k} & =\left.\left(\partial^{3} \varepsilon_{\mathbf{p}} / \partial p_{i} \partial p_{j} \partial p_{k}\right)\right|_{\mathbf{p}^{\star}} \\
z_{i j k} & =\left.\left(\partial^{3} \varepsilon_{\mathbf{p}} / \partial p_{i} \partial p_{j} \partial p_{k}\right)\right|_{\mathbf{p}^{\star}+\mathbf{Q}} .
\end{aligned}
$$

Equation 19 can be inverted to yield

$$
\delta \mathbf{p}=\mathbf{u}_{1} \varepsilon_{\mathbf{p}}+\mathbf{u}_{2} \varepsilon_{\mathbf{p}+\mathbf{Q}}=\left(\mathbf{u}_{1}+\frac{\Delta^{2}}{\varepsilon_{\mathbf{p}}^{2}} \mathbf{u}_{2}\right) \varepsilon_{\mathbf{p}}
$$

with

$$
\begin{aligned}
& \mathbf{u}_{1}=\frac{\mathbf{v}_{\mathbf{Q}}^{\star} \times\left[\mathbf{v}^{\star} \times \mathbf{v}_{\mathbf{Q}}^{\star}\right]}{\left(\mathbf{v}^{\star} \times \mathbf{v}_{\mathbf{Q}}^{\star}\right)^{2}} \\
& \mathbf{u}_{2}=\frac{\mathbf{v}^{\star} \times\left[\mathbf{v}_{\mathbf{Q}}^{\star} \times \mathbf{v}^{\star}\right]}{\left(\mathbf{v}^{\star} \times \mathbf{v}_{\mathbf{Q}}^{\star}\right)^{2}} .
\end{aligned}
$$

Differentiating Eq. (19), substituting Eq. 21) into it and using the result in Eq. (12) for the electrical conductivities, we obtain the linearized $T=0$ change in the electrical conductivity tensor $\delta \hat{\sigma}=\hat{\sigma}(\Delta)-\hat{\sigma}(\Delta=0)$ in multiples of the conductance quantum $\sigma_{Q}=e^{2} / \hbar$ as

$$
\begin{aligned}
& \delta \sigma_{x y}=\sigma_{Q} \tau^{2} \frac{B \Delta}{\Phi_{0}} \hat{\mathbf{z}} \cdot\left[\eta_{\mathbf{1}}^{\mathbf{p}}+\eta_{\mathbf{2}}^{\mathbf{s p}}+3 \eta_{\mathbf{2}}^{\mathbf{p}}+\mathbf{3} \eta_{\mathbf{1}}^{\mathbf{s p}}\right] \times\left(\mathbf{v}_{\mathbf{Q}}^{\star}-\mathbf{v}^{\star}\right) \\
& \delta \sigma_{x x}=-\sigma_{Q} \frac{\tau}{\pi} \frac{\left(\mathbf{v}^{\star}-\mathbf{v}_{\mathbf{Q}}^{\star}\right)^{2}}{\left|\mathbf{v}_{\mathbf{Q}}^{\star} \times \mathbf{v}_{\mathbf{Q}}^{\star}\right|} \Delta .
\end{aligned}
$$

Here, the vectors

$$
\begin{aligned}
\eta_{\mathbf{1}}^{\mathbf{p}} & =\left(m_{11} u_{1 x}+m_{12} u_{1 y}\right) \hat{\mathbf{x}}+\left(m_{21} u_{1 x}+m_{22} u_{1 y}\right) \hat{\mathbf{y}} \\
\eta_{\mathbf{2}}^{\mathbf{p}} & =\left(m_{11} u_{2 x}+m_{12} u_{2 y}\right) \hat{\mathbf{x}}+\left(m_{21} u_{1 x}+m_{22} u_{2 y}\right) \hat{\mathbf{y}} \\
\eta_{\mathbf{1}}^{\mathbf{p}+\mathbf{Q}} & =\left(n_{11} u_{1 x}+n_{12} u_{1 y}\right) \hat{\mathbf{x}}+\left(n_{21} u_{1 x}+n_{22} u_{1 y}\right) \hat{\mathbf{y}} \\
\eta_{\mathbf{2}}^{\mathbf{p}+\mathbf{Q}} & =\left(n_{11} u_{2 x}+n_{12} u_{2 y}\right) \hat{\mathbf{x}}+\left(n_{21} u_{1 x}+n_{22} u_{2 y}\right) \hat{\mathbf{y}}
\end{aligned}
$$

have been defined. Parenthetically, we note that the linearized change $\delta \sigma_{x x}$ in the electrical conductivity has been treated in great detail previously for the three dimensional SDW transition in Cr, with essentially the same result ${ }^{32}$. Via Eq. (13), changes in the thermoelectric conductivities are obtained from $\frac{d \delta \sigma_{i j}}{d \mu}$. These derivatives of Eq. 23) are obtained from the relations

$$
\begin{aligned}
\frac{d v_{i}^{\star}}{d \mu} & =\sum_{j} m_{i j}\left(u_{1}^{j}+u_{2}^{j}\right) \\
\frac{d m_{i j}}{d \mu} & =\sum_{k} v_{i j k}\left(u_{1}^{k}+u_{2}^{k}\right),
\end{aligned}
$$

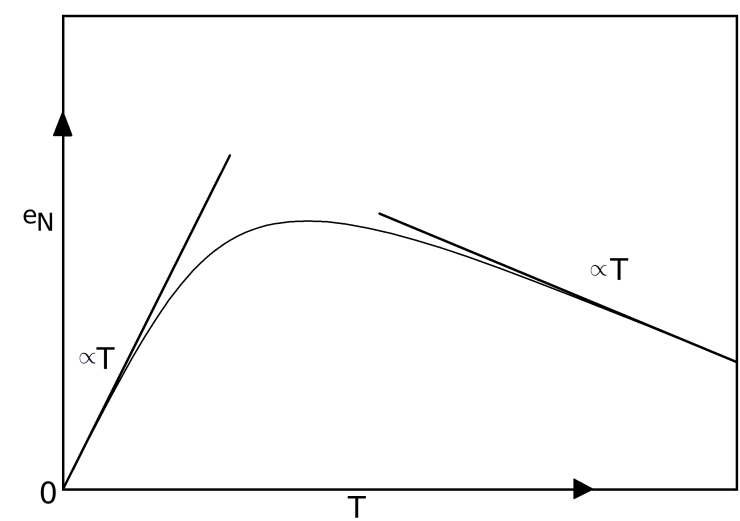

FIG. 4: Sketch of the normal state Nernst signal dependence on temperature. The linear temperature dependence at lowest $T$ turns over in a maximum at temperatures below the spin density wave ordering temperature. At optimal doping, the position of this maximum is roughly $50 \mathrm{~K}$. Above the peak temperature, the signal vanishes proportional to temperature.

Linearizing Eq. (11) in $\Delta$ in this way yields $\delta \vartheta_{x x}$ and $\delta \vartheta_{y x}$ to linear order in $\Delta$. From a numerical calculation of $\vartheta_{x x}$ and $\vartheta_{y x}$, we obtain the values $\delta \vartheta_{x x} / \vartheta_{x x}=47.4 \Delta$ and $\delta \vartheta_{y x} / \vartheta_{y x}=-39.8 \Delta$, see also Fig. 2. Very close to $x_{c}=0.165$ it might be difficult to measure the quantum critical contributions $\delta \vartheta_{y x}$ and $\delta \vartheta_{x x}$ experimentally due to other contributions to the signal which we could not specify.

\section{Finite temperatures}

At finite temperatures the Fermi surface as well as the quasiparticle scattering rate is expected to change. These effects will influence the temperature dependence of the Nernst signal which is sketched in Fig. 4. First of all, in cuprate materials it has to be considered that the quasiparticle scattering rate is linear in temperature in most parts of the Brillouin zone ${ }^{20}$. Important changes in the Fermi surface have to be considered at temperatures above $T_{S D W}$. Below this temperature, fluctuations of the SDW order parameter remain gapped and can be neglected. Once the SDW gap closes, the Fermi surface reconstructs and fluctuations of the SDW order parameter provide an important scattering mechanism. In the far underdoped region of electron doped cuprates, $T_{S D W}$ is of order the Debye temperature and scattering off phonons has to be considered as well.

In a range of temperatures above $T_{S D W}$, a sizable Nernst effect is still observed in experiment ${ }^{5}$. Our present mean-field theory for Fermi surface reconstruction cannot account for the size of the signals. However, there is no good theory for transport in this fluctuation regime. It would be interesting to examine the behavior in a recent theory of thermal fluctuations in the orientation of the SDW order ${ }^{39}$. The experimental observations at finite 
temperatures are not in contrast with our assumptions, which are only valid at temperatures below $T_{S D W}$, where fluctuations of the SDW order parameter are negligible. In fact, it was shown that antiferromagnetic fluctuations enhance the Nernst signal at finite temperatures above the magnetic ordering temperature $T_{S D W}$ and reproduce the peak structure in the normal state Nernst signal observed in experiment 31 . The relaxation time approximation (RTA) certainly fails to reproduce this effect, because it would predict a small Nernst signal above $T_{S D W}$. Above this temperature, the normal state quasiparticles are gapless and form a single carrier system. Within the relaxation time approximation, the Nernst signal is thus diminished by the Sondheimer cancellation 33 , in contrast to experimental results.

\section{ANTIFERROMAGNETIC FLUCTUATIONS}

Within our assumptions, the SDW gap vanishes at a quantum critical point upon doping with electron carriers. There has been substantial disagreement over the position of this quantum critical point. Elastic neutron scattering measurements on NCCO suggest short ranged antiferromagnetic order between $x=0.145$ and $x=0.154^{14}$, and it has been proposed that short ranged order might even occur at $x=0.134^{34}$. Transport measurements on PCCO show rapidly changing transport properties at dopings below $x=0.165$, suggesting that Fermi surface properties change drastically already slightly above optimal doping. Part of the confusion might originate from the uncertainty about the oxygen content of the samples, which makes it difficult to compare the effective carrier concentration of different samples.

Experimental results show a strong doping dependence of Hall and Nernst effect way above optimal doping 5 where clearly no spin density wave gap exists. This suggests that the band structure parameters change upon electron doping. One way to understand this behavior is to analyze self-energy corrections originating from antiferromagnetic spin-fluctuations. This analysis also helps to clarify whether short-ranged magnetic order can account for the observed Fermi surface reconstruction and enhancement of the normal state Nernst signal for electron dopings below $x \approx 0.16$.

The effect of spin fluctuations on the Fermi surface can be obtained from the real part of the electronic self energy. We neglect the imaginary part of the self energy by assuming again that impurity scattering dominates transport at lowest temperatures. As discussed in section III] scattering on spin flucuations would be beyond the scope of our approach due to the failure of the relaxation time approximation to treat this effect. The leading approximation to the electronic self energy due to spin fluctuations is

$\Sigma(\mathbf{k}, i \omega)=-g^{2} T \int d^{2} \mathbf{q} \sum_{i \Omega_{n}} G\left(\mathbf{k}+\mathbf{q}, i \omega+i \Omega_{n}\right) D\left(\mathbf{q}, i \Omega_{n}\right)$,

where $G$ and $D$ are electron and spin fluctuation Matsubara Green's functions, respectively. We will use: $G(\mathbf{p}, i \omega)=\left(i \omega-\zeta_{\mathbf{p}}\right)^{-1}$ and $D\left(\mathbf{q}, i \Omega_{n}\right)=-\left(\Gamma_{\mathbf{q}}+\left|\Omega_{n}\right|\right)^{-1}$, with $\zeta_{\mathbf{p}}=\varepsilon_{\mathbf{p}}-\mu$ and $\Gamma_{\mathbf{q}}=\Gamma\left(r+\xi^{2}(\mathbf{q}-\mathbf{Q})^{2}\right)$, where $\Gamma$ is the energy scale characteristic of spin fluctuations and $\xi$ the correlation length of magnetic order. The distance to the SDW quantum critical point is controlled by the parameter $r$, and the ordering wavevector is again $\mathbf{Q}=(\pi, \pi)$.

As has been discussed in Ref. 13, the self energy at $T=0$ and $i \omega=0$ can be integrated as

$$
\Sigma(\mathbf{k}, i \omega=0)=0.5 \lambda \zeta_{\mathbf{k}+\mathbf{Q}} \ln \left(r^{2}+\left(\zeta_{\mathbf{k}+\mathbf{Q}} / E_{0}\right)^{4}\right),
$$

where $r$ and $\zeta_{\mathbf{k}+\mathbf{Q}} / E_{0}$ are assumed to be small. The energy scale $E_{0}$ is a cutoff of order the normal state band width and $\lambda$ is a dimensionless coupling constant. The new electronic dispersion is

$$
\varepsilon_{\mathbf{p}}+\Sigma(\mathbf{p}, i \omega=0) .
$$

Using this new dispersion in Eqs (4) and (12), the resultant Nernst signal (not shown) is enhanced but negative for the renormalized Fermi surfaces shown in Fig. 5 . We obtained a negative Nernst signal for a wide range of parameters $r \in[0.001,0.1]$ and $\lambda \in[0.1,0.4]$, including a regime of very weak spin fluctuation effects where we believe Eq. (28) is an accurate approximation.

We conclude that the renormalization of the Fermi surface due to spin fluctuations (states without longrange SDW order) fails to reproduce the Nernst signal observed in experiment. At optimal doping and below, this means that the observed Fermi surface reconstruction is more likely to originate from long-range SDW order. This interpretation is also supported by a comparison of the renormalized Fermi surfaces in Fig. 5 with ARPES measurements on NCCO 7 . At electron dopings of $x=0.15$ and below, photoemission intensity is significantly suppressed near $(0.65 \pi, 0.3 \pi)$ (and its symmetry related points) at the intersection of the Fermi surface with the antiferromagnetic Brillouin zone boundary. This change in photoemission intensity cannot be explained from Eq. (28), since leading order self energy corrections are cancelled at any crossing point where $\varepsilon(\mathbf{p}+\mathbf{Q})=\varepsilon(\mathbf{p})=\mu$. Thus again, the opening of a SDW gap slightly above optimal doping $x=0.15$ seems more plausible to explain the Fermi surface reconstruction seen in experiment.

\section{CONCLUSIONS}

Our results show that SDW order in the electron-doped cuprates has fundamental implications for the Nernst signal and the thermopower. As the SDW gap becomes 


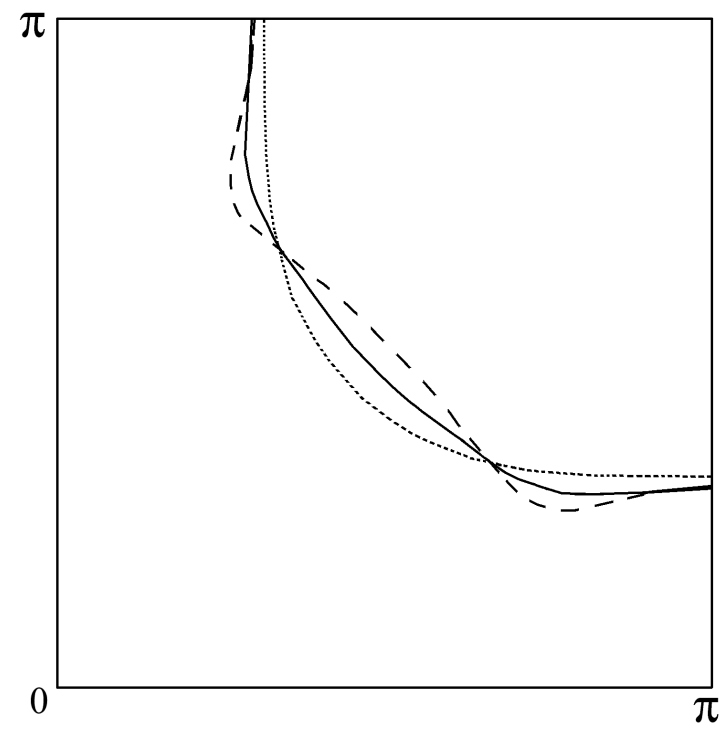

FIG. 5: Fermi surface changes due to finite spin correlation lengths corresponding to electron dopings somewhat above the SDW quantum critical point. The Fermi surface without any influences of spin fluctuations is shown as the dotted line. The dashed line shows a renormalized Fermi surface for a large coupling to spin fluctuations $(\lambda=0.4)$ and a small distance $r=0.001$ to the quantum critical point. Renormalization effects become weaker for a smaller coupling $\lambda=0.2$ and $r=0.1$ (continuous line). At the crossing points with $\varepsilon(\mathbf{p}+$ $\mathbf{Q})=\varepsilon(\mathbf{p})=\mu$, the Fermi surface remains unchanged by spin fluctuations. The cutoff energy is $E_{0}=0.88 \mathrm{eV}$.

stronger, the hole-like carriers will eventually vanish and the Nernst signal will have a large discontinuous change at the lowest $T$. This behavior is also obtained for the thermopower, where the discontinuity in addition should cause an observable sign change in the signal. At finite $T$, the discontinuities will be smeared out by thermally excited carriers and magnetic breakdown. To obtain our results, the presence of oppositely charged carriers represents a necessary, but not a sufficient condition in order to obtain an enhanced Nernst signal. The fundamental origin of the maximal Nernst signal within our calculation is a singularity in the quasiparticle density of states, while the Nernst signal gets weaker if the Fermi surface moves away from this singularity, although two types of carriers are still present in the Fermi surface. We note that the existence of oppositely charged and current carrying quasiparticles is a widespread argument to explain an enhanced normal state Nernst signal, but our results require a more subtle physical origin than the requirement of two types of carriers would represent.

In this sense, our results are also in contrast with the analysis of the ambipolar Nernst effect in Ref. 35, which predicts a maximal Nernst signal when hole and electronlike carrier densities exactly compensate each other. This explanation had been used previously to account for the large normal state Nernst signal in PCCO 5 . Within our analysis, the ambipolar signal is instead largest when the hole pockets just touch the Fermi surface, and decreases rapidly until the carriers compensate most.

Our findings are also likely of relevance to the holedoped cuprates. Recent explanations of a large normal state Nernst signal in these materials were based on the proposal of $d$-density wave order ${ }^{35 \mid 36}$. A large normal state Nernst signal has recently been reported ${ }^{38}$ in the stripe-ordered phase of $\mathrm{La}_{1.6-x} \mathrm{Nd}_{0.4} \mathrm{Sr}_{x} \mathrm{Cu}_{4}$, which vanished in the non-ordered state. These findings suggest that stripe order enhances the normal state Nernst effect, and it would be interesting to extend our results to spin/charge density wave orders.

The onset of "stripe" order, and the evolution from "large" to "small" Fermi surfaces with decreasing doping ${ }^{37}$ could lead to a large Nernst signal by the opening/closing of hole or electron pockets. The connection of such normal state features to those associated with the superconductor-insulator QPT computed earlier ${ }^{4}$ remains an important open problem, and some ideas have appeared in Ref. 39 .

In summary, we have presented a theory for the anomalously large normal state Nernst signal in the electrondoped cuprates. We established a direct relation between SDW order and the peak of the normal state Nernst signal at optimal doping. Finally, while the energy dependence of the scattering rate is unlikely to modify our result, a more detailed understanding of the scattering mechanism is necessary for a quantitative understanding of the large Nernst signal in the underdoped and overdoped regions.

We thank M. Müller, V. Galitski, R. Greene and L. Taillefer for useful discussions. L. Taillefer alerted us to observations ${ }^{37}$ connecting stripe order in the holedoped cuprates to a change in the Fermi surface. A. H. acknowledges support by the DFG through the SFB 608 (Köln) and the Research Units FG 538 and FG 960. The research was supported by the NSF under grant DMR0757145 and by the FQXi foundation.
1 Z. A. Xu, N. P. Ong, Y. Wang, T. Kakeshita, S. Uchida, Nature 406, 486 (2000); Y. Wang, L. Li, and N. P. Ong, Phys. Rev. B 73, 024510 (2006).

2 S. Mukerjee and D. A. Huse, Phys. Rev. B 70, 014506 (2004); D. Podolsky, S. Raghu, and A. Vishwanath, Phys. Rev. Lett. 99, 117004 (2007).
3 L. Balents, L. Bartosch, A. Burkov, S. Sachdev, and K. Sengupta, Phys. Rev. B 71, 144508 (2005).

4 S. A. Hartnoll, P. K. Kovtun, M. Müller,and S. Sachdev, Phys. Rev. B 76, 144502 (2007).

5 P. Li, and R. L. Greene, Phys. Rev. B 76, 174512 (2007).

${ }^{6}$ P. Fournier, X. Jiang, W. Jiang, S. N. Mao, T. Venkatesan, 
C. J. Lobb, and R. L. Greene, Phys. Rev. B 56, 14149 (1997).

7 N. P. Armitage, F. Ronning, D. H. Lu, C. Kim, A. Damascelli, K. M. Shen, D. L. Feng, H. Eisaki, and Z.-X. Shen, Phys. Rev. Lett. 88, 257001 (2002).

${ }^{8}$ H. Matsui, T. Takahashi, T. Sato, K. Terashima, H. Ding, T. Uefuji, and K. Yamada, Phys. Rev. B 75, 224514 (2007).

9 G. M. Luke, L. P. Le, B. J. Sternlieb, Y. J. Uemura, J. H. Brewer, R. Kadono, R. F. Kiefl, S. R. Kreitzman, T. M. Riseman, C. E. Stronach, M. R. Davis, S. Uchida, H. Takagi, Y. Tokura, Y. Hidaka, T. Murakami, J. Gopalakrishnan, A. W. Sleight, M. A. Subramanian, E. A. Early, J. T. Markert, M. B. Maple, and C. L. Seaman, Phys. Rev. B 42, 7981 (1990).

10 P. K. Mang, O. P. Vajk, A. Arvanitaki, J. W. Lynn, and M. Greven, Phys. Rev. Lett. 93, 027002 (2004).

11 P. Li, K. Behnia, and R. L. Greene, Phys. Rev. B 75, 020506 (2007).

12 Y. Dagan, M. M. Qazilbash, C. P. Hill, V. N. Kulkarni, and R. L. Greene, Phys. Rev. Lett. 92, 167001 (2004).

13 J. Lin, and A. J. Millis, Phys. Rev. B 72, 214506 (2005).

14 E. M. Motoyama, G. Yu, I. M. Vishik, O. P. Vajk, P. K. Mang, and M. Greven, Nature 445, 186-189 (2007).

15 O. K. Andersen, A. I. Liechtenstein, O. Jepsen, F. Paulsen, J. Phys. Chem. Solids 56, 1573 (1995).

16 G. Grüner, Rev. Mod. Phys. 66, 1 (1994).

17 In extended Hubbard models, several incommensurate order parameters can occur in different mean field theories. These can be associated with additional possibilities like charge density wave order or $d$-density wave order 18 . Such orders could possibly be realized in the hole-doped cuprates, while in the electron-doped cuprates, commensurate SDW order is the exclusive type of density wave order under consideration.

18 I. Dimov, P. Goswami, X. Jia, and S. Chakravarty, Phys. Rev. B 78, 134529 (2008).

19 Y. Onose, Y. Taguchi, K. Ishizaka, and Y. Tokura, Phys. Rev. Lett. 87, 217001 (2001).

20 T. Valla, A. V. Fedorov, P. D. Johnson, Q. Li, G. D. Gu, and N. Koshizuka, Phys. Rev. Lett. 85, 828 (2000).

21 E. I. Blount, Phys. Rev. 126, 1636 (1962).

22 X. J. Zhou, T. Yoshida, A. Lanzara, P. V. Bogdanov, S. A. Kellar, K. M. Shen, W. L. Yang, F. Ronning, T. Sasagawa, T. Kakeshita, T. Noda, H. Eisaki, et al.,
Nature 423, 398 (2003).

23 W. Yu, J. S. Higgins, P. Bach, and R. L. Greene, Phys. Rev. B 76, 020503(R) (2007).

24 J. M. Ziman, Electrons and Phonons (Oxford University Press, Oxford, 1960).

25 R. D. Barnard, Thermoelectricity in Metals and Alloys (Taylor \& Francis, London, 1972).

26 C. C. Homes, R. P. S. M. Lobo, P. Fournier, A. Zimmers, and R. L. Greene, Phys. Rev. B 74, 214515 (2006).

27 A. J. Millis, A. Zimmers, R. P. S. M. Lobo, N. Bontemps, and C. C. Homes, Phys. Rev. B 72, 224517 (2005).

${ }^{28}$ H. J. Kang, P. Dai, H. A. Mook, D. N. Argyriou, V. Sikolenko, J. W. Lynn, Y. Kurita, S. Komiya, and Y. Ando. Phys. Rev. B 71, 214512 (2005).

29 D. V. Livanov, Phys. Rev. B 60, 13439 (1999).

30 G. Baym and L. P. Kadanoff, Phys. Rev. 124, 287 (1961).

31 H. Kontani and K. Yamada, J. Phy. Soc. Jpn. 74 (2005) 155.

32 Y. B. Bazaliy, R. Ramazashvili, Q. Si, and M. R. Norman, Phys. Rev. B 69, 144423 (2004).

33 K. Behnia, J. Phys. C 21, 113101 (2009).

34 The identification of short-ranged antiferromagnetism (corresponding to a vanishing spin stiffness) at $x=0.134$ is only one possibility. As far as interpreted in Ref. 14, a non-zero spin stiffness at $x=0.134$ might fit the related experimental data as well. This way of fitting the experimental data leads to the interpretation that a vanishing spin-stiffness is observed only at dopings of $x=0.145$ or higher.

35 V. Oganesyan, and I. Ussishkin, Phys. Rev. B 70, 054503 (2004).

36 S. Tewari, and C. Zhang, arXiv:0902.0012

37 O. Cyr-Choinière, R. Daou, F. Laliberté, D. LeBoeuf, N. Doiron-Leyraud, J. Chang, J.-Q. Yan, J.-G. Cheng, J.S. Zhou, J.B. Goodenough, S. Pyon, T. Takayama, H. Takagi, Y. Tanaka, and L. Taillefer, Nature 458, 743 (2009).

38 R. Daou, N. Doiron-Leyraud, D. LeBoeuf, S. Y. Li, F. Laliberté, O. Cyr-Choinière, Y. J. Jo, L. Balicas, J.-Q. Yan, J.-S. Zhou, J. B. Goodenough, and L. Taillefer, Nature Phys. 5, 31 (2009).

39 V. Galitski and S. Sachdev, Phys. Rev. B 79, 134512 (2009). 